\title{
Impacts of Chemical Reaction, Diffusion-Thermo and Radiation on Unsteady Natural Convective Flow past an Inclined Vertical Plate under Aligned Magnetic Field
}

\author{
Suresh Babu Ramakrishna 1,* (D), Sravan Kumar Thavada 2 (D), Govindaraju Magge Venkatachala 1(D), \\ Mallikarjuna Bandaru ${ }^{3}$ iD
}

1 Department of Mathematics, M S Ramaiah Institute of Technology, Bangalore-560054, Karnataka, India; sureshbabu_r80@yahoo.co.in (S.B.R); govindarajumv@msrit.edu (G.M.V);

2 Department of Mathematics, REVA University, Bangalore-560064, Karnataka, India; thavadasravankumar@gmail.com (S.K.T);

3 Department of Mathematics, B.M.S College of Engineering, Bangalore-560004, Karnataka, India; mallikarjuna.jntua@gmail.com (M.B);

* Correspondence: sureshbabu_r80@yahoo.co.in;

Received: 6.01.2021; Revised: 1.02.2021; Accepted: 3.02.2021; Published: 8.02.2021

Abstract: In this article, diffusion-thermo, thermal radiation, and first-order chemical reaction effects are studied analytically when the aligned magnetic field set to the fluid/ the plate on the unsteady, free convective fluid passing through an inclined vertical plate by flexible surface conditions, concentration diffusion under the action of a coaxial magnetic field. The governing PDE's are derived from the physical model and transformed into dimensionless form. Then a closed-form solution is obtained using the Laplace transform method. The effects of controlling parametric quantities like M, R, Sc, Pr, Du, $\mathrm{Gr}, \mathrm{Gm}$ are analyzed through graphs for fluid properties. A comparative study has been made with published results in the absence of some non-dimensional parameters for a particular case (aligned magnetic field set to the fluid) found in good agreement.

Keywords: Aligned magnetic field; Dufour effect; Thermal radiation; Chemical reaction; Inclined plate; Laplace transforms method.

Nomenclature:

\begin{tabular}{l|l|l|l}
\hline$a^{\prime}$ & Absorption coefficient; & $G r$ & Thermal Grashof number; \\
\hline$T^{\prime}$ & Temperature of the fluid (K); & $R$ & Radiation parameter $\left(\mathrm{cm}^{-2}\right) ;$ \\
\hline$C^{\prime}$ & Species concentration; & $G m$ & Mass Grashof number; \\
\hline$T_{w}^{\prime}$ & Temperature of the plate; & $S c$ & Schmidt number; \\
\hline$C_{w}^{\prime}$ & Concentration of the plate; & $\mathrm{Pr}$ & Prandtl number; \\
\hline$T_{\infty}^{\prime}$ & Fluid temperature away from the plate; & $t$ & Dimensionless time (Sec); \\
\hline$C_{\infty}^{\prime}$ & Fluid Concentration away from the plate; & $N u$ & Nusselt number; \\
\hline$C^{\prime}$ & Dimensionless concentration $\left(\mathrm{kg} / \mathrm{m}^{3}\right) ;$ & $S h$ & Sherwood number; \\
\hline$C_{p}$ & Specific heat at constant pressure $(\mathrm{J} / \mathrm{kg} . \mathrm{K}) ;$ & $k$ & Chemical reaction $\left(\mathrm{W} / \mathrm{mK}^{3}\right) ;$ \\
\hline$C_{s}$ & Concentration susceptibility; & $\alpha$ & Angel of inclination; \\
\hline$\beta$ & Volumetric coefficient of thermal expansion; & $\mu$ & Coefficient of viscosity $\left(\mathrm{m}^{2} \mathrm{~s}^{-1}\right) ;$ \\
\hline$u_{0}$ & Velocity of the plate; & $v$ & Kinematic viscosity $\left(\mathrm{m}^{2} \mathrm{~s}^{-1}\right) ;$ \\
\hline$B_{0}$ & External aligned magnetic field $\left(\mathrm{A} \cdot \mathrm{m}^{2}\right) ;$ & $\sigma$ & Electric conductivity $\left(\mathrm{Sm}{ }^{-1}\right) ;$ \\
\hline$q_{r}$ & Radiative heat flux; & $\theta$ & Dimensionless temperature $(\mathrm{K}) ;$ \\
\hline
\end{tabular}




\begin{tabular}{l|l|l|l}
\hline$\rho$ & Density of the fluid $\left(\mathrm{kgm}^{-3}\right) ;$ & erf & Error function; \\
\hline$M$ & Magnetic field parameter $\left(\mathrm{Am}^{-1}\right) ;$ & erfc & Complementary error function; \\
\hline$D_{m}$ & Coefficient of mass diffusivity; & & Subscripts \\
\hline$\beta^{*}$ & Volumetric coefficient of concentration; & $w$ & Conditions on the wall; \\
\hline$g$ & Acceleration $\left(\mathrm{m} / \mathrm{s}^{2}\right) ;$ & $\infty$ & Freestream conditions; \\
\hline$D_{u}$ & Diffusion-thermo effect; & & \\
\hline
\end{tabular}

(C) 2021 by the authors. This article is an open-access article distributed under the terms and conditions of the Creative Commons Attribution (CC BY) license (https://creativecommons.org/licenses/by/4.0/).

\section{Introduction}

Natural or free convection is a random flow resulting from non-homogeneous fields of volumetric forces like Coriolis, MHD, gravitational, centrifugal, etc. Various researchers have been studied this phenomenon. Free/Natural convection flow has numerous practical applications and environmental situations such as chilling of electronic machinery, geothermal systems, material processing, designs connected to thermal insulation, the security of energy systems, atmospheric flows, air conditioning systems etc. Also, heat transfer applications by moving material in a moving fluid medium have a wide range of real-world applications. There are some flows on the earth whose rate is made not only by the temperature differences but also by the concentration variations. In atmosphere science, where variations among land and air temperatures can increase to complex flow shapes, buoyancy is also significant. The study of the combined transfer of heat and mass on free convection received considerable interest in many theoretical models and experimental/practical aspects because of its various applications in industry, scientific, and engineering processes. In the literature, such problems are dealt with the Newtonian/non-Newtonian fluids for many geometries such as elliptical, rectangular or cube, triangular and circular cylinders with various boundary conditions and were used different techniques such as computational, theoretical, and experimental approaches. An electrically conducting fluid flow with an external constraint of the magnetic field can be regulated. The transfer rate can also be controlled. Various industrial applications can be seen in many sciences and technology, viz nuclear cooling reactors, boundary layer control in aerodynamics, plasma studies, petroleum industries, crystal growth, etc. Hence, authors are received new attention for the study of most general contexts of MHD with the influence of the external force of the magnetic field on electrically conduction fluid.

Many practical applications like Hall accelerators, MHD power generators etc., in the engineering and industrial point of view, authors have given a considerable interest in studying Soret and Dufour effects along MHD flows when heat mass transfer occurs concurrently in moving fluids. Kao et al. [1] examined the solution heat transfer response of a free convective flow along with a flat plate with wall temperature discontinuity. For the plate's impulsive and uniformly accelerated motion, Rapits et.al. [2] studied the influence of an unvarying moving magnetic field of an electrically conducting fluid. Tokis et al. [3] studied the influence of a uniform moving magnetic field fixed to the fluid. Prasad et al. [4] have performed the effects of temperature transport properties on completely developed MHD free convective flow by considering viscous and Ohmic dissipation. On the forced convective heat transfer, Sheikholeslami et al. [5] investigated the outcome of a non-uniform magnetic field in a liddriven half annuluses closure packed with $\mathrm{Fe}_{3} \mathrm{O}_{4}$-water Nanofluid. The fluid's magnetization is presumed to differ linearly with the strength of the temperature and magnetic field. Rashidi et al. [6] studied the heat transfer and hydrodynamic characteristics on the mixed convective 
nanofluid flow with sinusoidal walls under a magnetic field numerically. Shateyi et al. [7] examined MHD natural convective heat mass transfer flow under thermal radiation and chemical reaction over a permeable moving vertical plate with a convective boundary state using the spectral relaxation method. Khadijah et al. [8] analyzed normal convective viscous fluid in an annulus by ramped boundary flow based on time-dependent magnetohydrodynamic. This analysis aims to increase natural convective heat transfer, a zigzag-shaped ribs applied to the vertical, isothermal, heated surfaces. Ilias et al. [9] done theoretical research on the unstable aligned MHD boundary layer heat transfer nanofluid flow through an inclined plate at the interior edge. Fenuga et al. [10] studied the mathematical model and its solution on unstable fourth-grade MHD fluid flow using a Homotopy perturbation system under the magnetic field and suction/injection action. Prasad et al. [11] examined thermal radiation and absorption effects for a Kuvshinski fluid model with chemical reaction under an aligned magnetic field on the unsteady MHD flow. Effect of aligned magnetic field analyzed by Bilal et al. [12] for an upper convicted Maxwell fluid over an inclined stretching sheet.

Most of the authors have shown their keen interest in the combined effects of diffusionthermo and thermo-diffusion because of its various applications in many engineering fields. In most studies, Soret and Dufour's effects are ignored due to the small magnitude of order on fluid behavior. But, in reality, Soret and Dufour's effects play a significant role when the density variations occur for the moving fluids in areas such as geosciences, petrology, hydrology etc.. They are more critical and interesting macroscopically because of their physical phenomenon in fluid mechanics. Alam et al. [13] have studied the effects of Dufour and Soret of a flat plate. Babu et al. [14] conducted a numerical study for Soret and Dufour's effects on the mixed convective electrically conducting heat transfer flow with varying fluid properties under the magnetic field. The diffusion-thermo effect is studied by Reddy et al. [15] in a theoretical way on the heat mass transfer flow of a viscous fluid with the aligned magnetic field. Rao et al. [16] published thermochemical diffusion and thermal diffusion effects using Galerkin finite element analysis on the mass and heat transfer flux.

In fluid dynamics, thermal radiation is one of the essential topics of the engineering sciences, especially in aerospace, mechanical, chemical, environmental etc. and radiated heat transfer flow plays a vital role in manufacturing industries such as wings production, steel rolling, gas turbines, nuclear reactors, various aircraft devices, furnace design, materials processing, remote sensing for astronomy, temperature measurements, food processing and numerous agricultural, as well as cryogenic engineering applications. Thermal radiation is studied numerically with various effects by (a) Rao et al. [17] on unsteady MHD Casson fluid from a vertical porous plate; (b) Mahato et al. [18] on MHD heat-absorbing unsteady Casson fluid past a flat plate; (c) Ekakitie et al. [19] on MHD free convection flow over an inclined plate, and (d) Lavanya et al. [20] on unsteady MHD viscoelastic flow past a tilted plate. In industrial and engineering sciences, under chemical reaction, combined heat and mass transfer flows have a finite number of applications like enhanced oil recovery, geothermal reservoirs, fibrous insulation, drying of porous solids, and polymers cooling and nuclear reactors, oxidation, and synthesis materials, thermal insulation etc. The study of MHD flow with an external constraint of the magnetic field can be regulated. The transfer rate can also be controlled. With this point of view, various industrial applications can be seen in many sciences and technology branches. Hence, the authors received new attention for the study of most general context of MHD with the influence of the magnetic field's external force on electrically conduction fluid. Such a MHD study has been studied for various fluids numerically under the 
radiation and chemical reaction effects along with the other effects, by (a) Babu et al. [21] varying liquid properties on the mixed convective flow from the vertical plate, (b) Krishna et al. [22] on the Sakiadis and the Blasius flow over a stationary flat plate, (c) Lalitha et al. [23] on the Oldroyd fluid flow along with a moving vertical plate, (d) Babu et al. [24] on the mixed convective flow past a vertical plate in a sparsely packed medium, (e) Endalew et al. [25] on the unstable natural convective flow past an oscillating slanted plate, (f) Sarma et al. [26] on the laminar mixed free-forced convective flow over a plate under a moving magnetic field, (g) Mohan et al. [27] on free convective Casson fluid flow past an exponentially vertical plate, (h) Omamoke et al. [28] on free convective flow over an inclined plate using perturbation technique by viscous dissipation and heat source, (i) Raju et al. [29] tested the convergence and grid independence analysis of an electrically conducting fluid past a moving plate, (j) Rao et al. [30] and Rama et al. [31] on free convective Casson fluid flow past vertical plate under the heat source. Omamoke et al. [32] have examined chemical reactions and thermal radiation effects on unstable blood flow through a parallel and horizontal plate in a porous and saturated medium with an inclined magnetic field.

The above literature is the motivation behind the main emphasis in studying the chemical reaction, Dufour, and radiation effects on unsteady MHD natural convective flow past an inclined plate subject to an adjustable temperature and mass diffusion under the applied aligned magnetic field. This problem is discussed in two cases when the aligned magnetic field imposed relative (i) to the fluid and (ii) to the plate. To find an exact solution of the physical model, the governed PDE's are solved using the Laplace transform technique and the boundary conditions.

\section{Mathematical Formulation}

Consider an unsteady, free convective, incompressible electrically conducting and radiated viscous fluid passing through an impulsively inclined plate by variable surface conditions and concentration diffusion under a uniform moving aligned magnetic field when the aligned magnetic field imposed to the fluid or the plate. Also, diffusion-thermo and firstorder chemical reaction are taken into account. A uniform aligned magnetic field $B_{0}$ is imposed in the $y^{\prime}$-direction. The $x^{\prime}$-axis is taken along the plate facing up, and the $y^{\prime}$-axis is taken perpendicular (normal) to the imposed aligned magnetic field. Further, it is assumed that the plate and the surrounding fluid have the same surface condition and have the same concentration at all the points in the total flow area $y^{\prime} \geq 0$. The plate has a constant speed of $u=u_{0} \exp \left(a^{\prime} t^{\prime}\right)$ and the state of the plate surface increases linearly with time $t$. The mass levels around the plate increases to $C_{w}^{\prime}$ at $t^{\prime} \geq 0$.

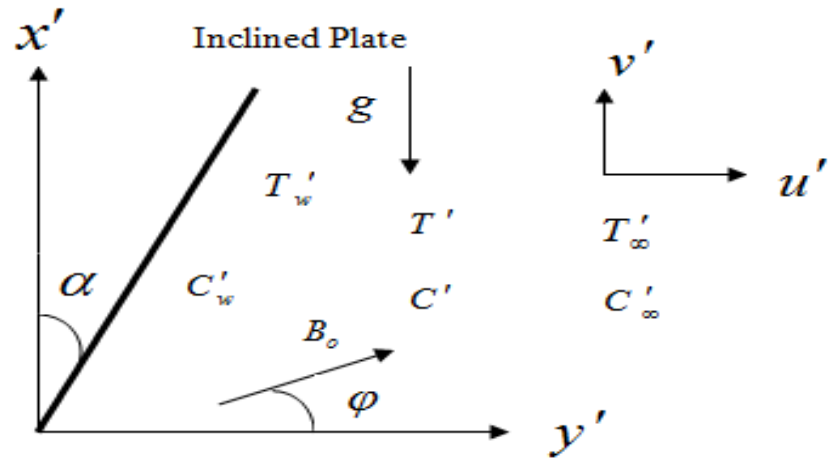

Figure 1. Geometry of the problem. 
The following assumptions are made to develop a mathematical model for the free convective motion: (i) the induced magnetic field is not taken into account due to very low magnetic Reynolds number when comparing the imposed magnetic field; (ii) the viscous dissipation is ignored in the energy equation; (iii) in Boussinesque's approximation, the effects of density fluctuations and species concentration are taken into account; (iv) a thin liquid is considered with absorbing/emitting radiation in the form of a non-scattering medium; (v) all quantities are functions of $y^{\prime}$ and $t^{\prime}$ only.

The flow equations and their associated conditions, when $B_{0}$ fixed to an accelerated plate, are as follows:

$$
\begin{aligned}
& \frac{\partial u^{\prime}}{\partial t^{\prime}}=v \frac{\partial^{2} u^{\prime}}{\partial y^{\prime 2}}+g \beta^{*}\left(C^{\prime}-C_{\infty}^{\prime}\right) \cos \alpha+g \beta\left(T^{\prime}-T_{\infty}^{\prime}\right) \cos \alpha-\frac{\sigma B_{0}^{2}}{\rho}\left[\sin ^{2} \varphi u^{\prime}-K u_{0} \exp \left(a_{0}^{\prime} t^{\prime}\right)\right], \\
& \frac{\partial T^{\prime}}{\partial t^{\prime}}=\frac{k}{\rho c_{p}} \frac{\partial^{2} T^{\prime}}{\partial t^{\prime 2}}+\frac{D_{m} K_{T} \rho}{\rho c_{p} C_{s}} \frac{\partial^{2} C^{\prime}}{\partial y^{\prime 2}}-\frac{1}{\rho c_{p}} \frac{\partial q_{r}}{\partial y}, \\
& \frac{\partial C^{\prime}}{\partial t^{\prime}}=D \frac{\partial^{2} C^{\prime}}{\partial y^{\prime 2}}-k^{\prime}\left(C^{\prime}-C_{\infty}^{\prime}\right), \\
& t^{\prime} \leq 0: u^{\prime}=0, \quad T^{\prime}=T_{\infty}^{\prime}, \quad C^{\prime}=C_{\infty}^{\prime}, \text { for all } y^{\prime}, \\
& t^{\prime}>0: u^{\prime}=u_{0} \exp \left(a^{\prime} t^{\prime}\right), T^{\prime}=T_{\infty}^{\prime}+\left(T_{w}^{\prime}-T_{\infty}^{\prime}\right) A t^{\prime}, C^{\prime}=C_{\infty}^{\prime}+\left(C_{w}^{\prime}-C_{\infty}^{\prime}\right) A t^{\prime}, \text { at } y^{\prime}=0, \\
& \quad u^{\prime}=0, T^{\prime} \rightarrow T_{\infty}^{\prime}, C^{\prime} \rightarrow C_{\infty}^{\prime}, \text { as } y^{\prime} \rightarrow \infty .
\end{aligned}
$$

Here, $K=0,1$ represents when $B_{0}$ set to the fluid or the plate respectively, $a_{0}^{\prime}$ is dimensional accelerating parameter, and $A=\frac{u_{0}^{2}}{v}$.

The local radiant by Rosseland $[19,23,28]$ approximation is for an optical thin gray gas is given by

$$
\frac{\partial q_{r}}{\partial y^{\prime}}=-4 a^{*} \sigma\left(T_{\infty}^{\prime 4}-T^{\prime 4}\right) .
$$

Substituting equation (5) in (2) and rewrite, we get

$$
\frac{\partial T^{\prime}}{\partial t^{\prime}}=\frac{k}{\rho c_{p}} \frac{\partial^{2} T^{\prime}}{\partial t^{\prime 2}}+\frac{D_{m} K_{T} \rho}{\rho c_{p} C_{s}} \frac{\partial^{2} C^{\prime}}{\partial y^{\prime 2}}-\frac{16 a^{*} \sigma T_{\infty}^{\prime 3}\left(T_{\infty}^{\prime}-T^{\prime}\right)}{\rho c_{p}},
$$

Introducing dimensionless quantities to express (1), (3), and (6) in non-dimensional form

$$
\begin{aligned}
& u=\frac{u^{\prime}}{u_{0}}, \theta=\frac{T^{\prime}-T_{\infty}^{\prime}}{T_{w}^{\prime}-T_{\infty}^{\prime}}, \phi=\frac{C^{\prime}-C_{\infty}^{\prime}}{C_{w}^{\prime}-C_{\infty}^{\prime}}, S c=\frac{v}{D}, \quad y=\frac{y^{\prime} u_{0}}{v}, G r=\frac{g \beta v\left(T_{w}^{\prime}-T_{\infty}^{\prime}\right)}{u_{0}^{3}}, \operatorname{Pr}=\frac{\mu C_{\rho}}{k}, t=\frac{t^{\prime} u_{0}^{2}}{v}, \\
& M=\frac{\sigma B_{0}^{2} v}{\rho u_{0}^{2}}, R=\frac{16 a^{*} v^{2} \sigma T_{\infty}^{\prime 3}}{k u_{0}^{2}}, k=\frac{v k^{\prime}}{u_{0}^{2}}, G m=\frac{g \beta^{*} v\left(C_{w}^{\prime}-C_{\infty}^{\prime}\right)}{u_{0}^{3}}, D u=\frac{D_{m} K_{T}\left(C_{w}^{\prime}-C_{\infty}^{\prime}\right)}{c_{s} c_{p} v\left(T_{w}^{\prime}-T_{\infty}^{\prime}\right)} .
\end{aligned}
$$

The resultant PDE's and associated conditions in the dimensionless form are:

$$
\begin{aligned}
& \frac{\partial u}{\partial t}=\frac{\partial^{2} u}{\partial y^{2}}+G m \phi \cos \alpha+G r \theta \cos \alpha-M\left(\sin ^{2} \varphi u-K e^{a_{0} t}\right), \\
& \frac{\partial \theta}{\partial t}=\frac{1}{\operatorname{Pr}} \frac{\partial^{2} \theta}{\partial y^{2}}-\frac{R}{\operatorname{Pr}} \theta+D u \frac{\partial^{2} \phi}{\partial y^{2}}, \\
& \frac{\partial \phi}{\partial t}=\frac{1}{S c} \frac{\partial^{2} \phi}{\partial y^{2}}-k \phi, \\
& t \leq 0: u=0, \theta=0, \phi=0, \text { for all } y,
\end{aligned}
$$


$t>0: u=e^{a_{0} t}, \theta=t, \phi=t$, at $y=0$,

$$
u \rightarrow 0, \theta \rightarrow 0, \phi \rightarrow 0 \text { as } y \rightarrow \infty \text {. }
$$

The dimensionless PDE's (8)-(10) are solved along the BC's (11) by Laplace transform technique and finally expressed the solutions are in exponential form as follows

$$
\begin{aligned}
& u(y, t)=\frac{\exp \left(a_{0} t\right)}{2}\left[\begin{array}{rl}
\exp \left(y \sqrt{M_{1}+a_{0}}\right) \operatorname{erfc}\left(\frac{y}{2 \sqrt{t}}+\sqrt{\left(M_{1}+a_{0}\right)} t\right) \\
\left.+\exp \left(-y \sqrt{M_{1}+a_{0}}\right) \operatorname{erfc}\left(\frac{y}{2 \sqrt{t}}-\sqrt{\left(M_{1}+a_{0}\right)} t\right)\right]
\end{array}\right. \\
& +A_{1}\left[\left(\frac{t}{2}+\frac{y}{4 \sqrt{M_{1}}}\right) \exp \left(y \sqrt{M_{1}}\right) \operatorname{erfc}\left(\frac{y}{2 \sqrt{t}}+\sqrt{M_{1} t}\right)\right. \\
& \left.+\left(\frac{t}{2}-\frac{y}{4 \sqrt{M_{1}}}\right) \exp \left(-y \sqrt{M_{1}}\right) \operatorname{erfc}\left(\frac{y}{2 \sqrt{t}}-\sqrt{M_{1} t}\right)\right] \\
& +\frac{A_{4}}{2} \exp \left(a_{2} t\right)\left[\exp \left(y \sqrt{M_{1}+a_{2}}\right) \operatorname{erfc}\left(\frac{y}{2 \sqrt{t}}+\sqrt{\left(M_{1}+a_{2}\right) t}\right)\right. \\
& \left.+\exp \left(-y \sqrt{M_{1}+a_{2}}\right) \operatorname{erfc}\left(\frac{y}{2 \sqrt{t}}-\sqrt{\left(M_{1}+a_{2}\right)} t\right)\right] \\
& +\frac{A_{2}}{2}\left[\exp \left(y \sqrt{M_{1}}\right) \operatorname{erfc}\left(\frac{y}{2 \sqrt{t}}+\sqrt{M_{1} t}\right)+\exp \left(-y \sqrt{M_{1}}\right) \operatorname{erfc}\left(\frac{y}{2 \sqrt{t}}-\sqrt{M_{1} t}\right)\right] \\
& -\frac{A_{5}}{2} \exp \left(a_{12} t\right)\left[\begin{array}{r}
\exp \left(y \sqrt{M_{1}+a_{12}}\right) \operatorname{erfc}\left(\frac{y}{2 \sqrt{t}}+\sqrt{\left(M_{1}+a_{12}\right) t}\right) \\
\left.+\exp \left(-y \sqrt{M_{1}+a_{12}}\right) \operatorname{erfc}\left(\frac{y}{2 \sqrt{t}}-\sqrt{\left(M_{1}+a_{12}\right)} t\right)\right]
\end{array}\right. \\
& +\frac{A_{3}}{2} \exp \left(-a_{9} t\right)\left[\exp \left(y \sqrt{M_{1}-a_{9}}\right) \operatorname{erfc}\left(\frac{y}{2 \sqrt{t}}+\sqrt{\left(M_{1}-a_{9}\right)} t\right)\right. \\
& \left.+\exp \left(-y \sqrt{M_{1}-a_{9}}\right) \operatorname{erfc}\left(\frac{y}{2 \sqrt{t}}-\sqrt{\left(M_{1}-a_{9}\right) t}\right)\right] \\
& +\frac{A_{6}}{2} \exp \left(-a_{11} t\right)\left[\begin{array}{r}
\exp \left(y \sqrt{M_{1}-a_{11}}\right) \operatorname{erfc}\left(\frac{y}{2 \sqrt{t}}+\sqrt{\left(M_{1}-a_{11}\right) t}\right) \\
\left.+\exp \left(-y \sqrt{M_{1}-a_{11}}\right) \operatorname{erfc}\left(\frac{y}{2 \sqrt{t}}-\sqrt{\left(M_{1}-a_{11}\right) t}\right)\right]
\end{array}\right. \\
& -\frac{A_{7}}{2} \exp \left(a_{0} t\right)\left[\exp \left(y \sqrt{M_{1}+a_{0}}\right) \operatorname{erfc}\left(\frac{y}{2 \sqrt{t}}+\sqrt{\left(M_{1}+a_{0}\right)} t\right)\right. \\
& \left.+\exp \left(-y \sqrt{M_{1}+a_{0}}\right) \operatorname{erfc}\left(\frac{y}{2 \sqrt{t}}-\sqrt{\left(M_{1}+a_{0}\right)}\right)\right] \\
& +\frac{A_{9}}{2}\left[\exp (y \sqrt{R}) \operatorname{erfc}\left(\frac{y \sqrt{\operatorname{Pr}}}{2 \sqrt{t}}+\sqrt{\frac{R t}{\operatorname{Pr}}}\right)+\exp (-y \sqrt{R}) \operatorname{erfc}\left(\frac{y \sqrt{\operatorname{Pr}}}{2 \sqrt{t}}-\sqrt{\frac{R t}{\operatorname{Pr}}}\right)\right] \\
& -A_{11}\left[\begin{array}{r}
\left(\frac{t}{2}+\frac{y \sqrt{S c}}{4 \sqrt{k}}\right) \exp (y \sqrt{k S c}) \operatorname{erfc}\left(\frac{y \sqrt{S c}}{2 \sqrt{t}}+\sqrt{k t}\right) \\
+\left(\frac{t}{2}-\frac{y \sqrt{S c}}{4 \sqrt{k}}\right) \exp (-y \sqrt{k S c}) \operatorname{erfc}\left(\frac{y \sqrt{S c}}{2 \sqrt{t}}-\sqrt{k t}\right)
\end{array}\right] \\
& +\frac{A_{13}}{2} \exp \left(-a_{11} t\right)\left[\begin{array}{r}
\exp \left(y \sqrt{\left(k-a_{11}\right) S c}\right) \operatorname{erfc}\left(\frac{y \sqrt{S c}}{2 \sqrt{t}}+\sqrt{\left(k-a_{11}\right) t}\right) \\
+\exp \left(-y \sqrt{\left(k-a_{11}\right) S c}\right) \operatorname{erfc}\left(\frac{y \sqrt{S c}}{2 \sqrt{t}}-\sqrt{\left(k-a_{11}\right) t}\right)
\end{array}\right]
\end{aligned}
$$




$$
\begin{aligned}
& +\frac{A_{10}}{2} \exp \left(-a_{9} t\right)\left[\begin{array}{r}
\exp \left(y \sqrt{R-a_{9}} \operatorname{Pr}\right) \operatorname{erfc}\left(\frac{y \sqrt{\operatorname{Pr}}}{2 \sqrt{t}}+\sqrt{-a_{9} t+\frac{R t}{\operatorname{Pr}}}\right) \\
\left.+\exp \left(-y \sqrt{R-a_{9}} \operatorname{Pr}\right) \operatorname{erfc}\left(\frac{y \sqrt{\operatorname{Pr}}}{2 \sqrt{t}}-\sqrt{-a_{9} t+\frac{R t}{\operatorname{Pr}}}\right)\right],
\end{array}\right] \\
& \theta(y, t)=\left(1+a_{5}\right)\left[\begin{array}{r}
\left(\frac{t}{2}+\frac{y \operatorname{Pr}}{4 \sqrt{R}}\right) \exp (y \sqrt{R}) \operatorname{erfc}\left(\frac{y \sqrt{\operatorname{Pr}}}{2 \sqrt{t}}+\sqrt{\frac{R t}{\operatorname{Pr}}}\right) \\
+\left(\frac{t}{2}-\frac{y \operatorname{Pr}}{4 \sqrt{R}}\right) \exp (-y \sqrt{R}) \operatorname{erfc}\left(\frac{y \sqrt{\operatorname{Pr}}}{2 \sqrt{t}}-\sqrt{\frac{R t}{\operatorname{Pr}}}\right)
\end{array}\right] \\
& +\frac{a_{7}}{2} \exp \left(a_{2} t\right)\left[\begin{array}{r}
\exp \left(y \sqrt{R+a_{2} \operatorname{Pr}}\right) \operatorname{erfc}\left(\frac{y \sqrt{\operatorname{Pr}}}{2 \sqrt{t}}+\sqrt{a_{2} t+\frac{R t}{\operatorname{Pr}}}\right) \\
+\exp \left(-y \sqrt{R+a_{2} \operatorname{Pr}}\right) \operatorname{erfc}\left(\frac{y \sqrt{\operatorname{Pr}}}{2 \sqrt{t}}-\sqrt{a_{2} t+\frac{R t}{\operatorname{Pr}}}\right)
\end{array}\right] \\
& -a_{5}\left[\left(\frac{t}{2}+\frac{y \sqrt{S c}}{4 \sqrt{k}}\right) \exp (y \sqrt{k S c}) \operatorname{erfc}\left(\frac{y \sqrt{S c}}{2 \sqrt{t}}+\sqrt{k t}\right)\right. \\
& \left.+\left(\frac{t}{2}-\frac{y \sqrt{S c}}{4 \sqrt{k}}\right) \exp (-y \sqrt{k S c}) \operatorname{erfc}\left(\frac{y \sqrt{S c}}{2 \sqrt{t}}-\sqrt{k t}\right)\right] \\
& \phi(y, t)=\left[\begin{array}{r}
\left(\frac{t}{2}+\frac{y \sqrt{S c}}{4 \sqrt{k}}\right) \exp (y \sqrt{k S c}) \operatorname{erfc}\left(\frac{y \sqrt{S c}}{2 \sqrt{t}}+\sqrt{k t}\right) \\
+\left(\frac{t}{2}-\frac{y \sqrt{S c}}{4 \sqrt{k}}\right) \exp (-y \sqrt{k S c}) \operatorname{erfc}\left(\frac{y \sqrt{S c}}{2 \sqrt{t}}-\sqrt{k t}\right)
\end{array}\right] .
\end{aligned}
$$

Where, $a_{1}=\frac{-D u \operatorname{Pr}}{S c-\operatorname{Pr}}, a_{2}=\frac{R-k S c}{S c-\operatorname{Pr}}, a_{3}=\frac{a_{1} S c}{a_{2}}, a_{4}=\frac{a_{1} k S c}{a_{2}^{2}}, a_{5}=\frac{a_{1} k S c}{a_{2}}, a_{6}=\left(1+a_{5}\right)$, $a_{7}=\left(a_{3}+a_{4}\right), a_{8}=\frac{G r \cos \alpha}{\operatorname{Pr}-1}, a_{9}=\frac{R-M_{1}}{\operatorname{Pr}-1}, a_{10}=\frac{G r \cos \alpha}{S c-1}, a_{11}=\frac{k S c-M_{1}}{S c-1}, a_{12}=\frac{G m \cos \alpha}{S c-1}$, $a_{13}=\frac{a_{8}}{a_{9}}, a_{14}=\frac{a_{8}}{a_{9}^{2}}, a_{15}=\frac{a_{8} a_{1} S c}{a_{2} a_{9}}, a_{16}=\frac{a_{1} a_{8} S c}{a_{2}\left(a_{2}+a_{9}\right)}, a_{17}=\frac{a_{1} a_{8} S c}{a_{9}\left(a_{2}+a_{9}\right)}, a_{18}=\frac{a_{8} a_{1} k S c}{a_{2} a_{9}}$, $a_{19}=\frac{a_{1} a_{8}\left(a_{2}-a_{9}\right) k S c}{a_{2}^{2} a_{9}^{2}}, a_{20}=\frac{a_{8} a_{1} k S c}{a_{2}^{2}\left(a_{2}+a_{9}\right)}, a_{21}=\frac{a_{8} a_{1} k S c}{a_{9}^{2}\left(a_{2}+a_{9}\right)}, a_{22}=\frac{a_{10} a_{1} S c}{a_{11} a_{12}}, a_{23}=\frac{a_{10} a_{1} S c}{a_{12}\left(a_{11}+a_{12}\right)}$, $a_{24}=\frac{a_{10} a_{1} S c}{a_{11}\left(a_{11}+a_{12}\right)}, a_{25}=\frac{a_{1} a_{10} k S c}{a_{11} a_{12}}, a_{26}=\frac{a_{1} a_{10} k S c\left(a_{12}-a_{11}\right)}{a_{11}^{2} a_{12}^{2}}, a_{27}=\frac{a_{10} a_{1} k S c}{a_{12}^{2}\left(a_{11}+a_{12}\right)}, a_{28}=\frac{a_{10} a_{1} k S c}{a_{11}^{2}\left(a_{11}+a_{12}\right)}$, $a_{29}=\frac{a_{12}}{a_{11}}, a_{30}=\frac{a_{12}}{a_{11}^{2}}, a_{31}=\frac{M K}{M_{1}+a_{0}}, A_{1}=\left(a_{13}-a_{18}+a_{25}+a_{29}\right), A_{2}=\left(-a_{14}-a_{15}+a_{19}+a_{22}-a_{26}-a_{30}\right)$, $A_{3}=\left(a_{14}+a_{17}-a_{21}\right), A_{4}=\left(a_{16}+a_{20}\right), A_{5}=\left(a_{23}+a_{27}\right), A_{6}=\left(-a_{24}+a_{28}+a_{30}\right), A_{7}=a_{31}$, $A_{8}=\left(-a_{13}+a_{18}\right), A_{9}=\left(a_{14}+a_{15}-a_{19}\right), M_{1}=M \operatorname{Sin}^{2} \varphi \quad A_{10}=\left(-a_{14}-a_{17}+a_{21}\right), A_{11}=\left(a_{25}+a_{29}\right)$, $A_{12}=\left(-a_{22}+a_{26}+a_{30}\right), A_{13}=\left(a_{24}-a_{28}-a_{30}\right)$.

The Nusselt and Sherwood number's in the non-dimensional form are given by:

$$
\begin{aligned}
& N u=a_{6}\left[t \sqrt{R} \operatorname{erf} \sqrt{\frac{R t}{\operatorname{Pr}}}+\sqrt{\frac{t \operatorname{Pr}}{\pi}} \exp \left(-\frac{R t}{\operatorname{Pr}}\right)+\frac{\operatorname{Pr}}{2 \sqrt{R}} \operatorname{erf} \sqrt{\frac{R t}{\operatorname{Pr}}}\right] \\
& -\left[\sqrt{\frac{\operatorname{Pr}}{\pi t}} \exp \left(-\frac{R t}{\operatorname{Pr}}\right)+\sqrt{R} \operatorname{erf} \sqrt{\frac{R}{\operatorname{Pr}} t}\right]+a_{7}\left[\sqrt{\frac{S c}{\pi t}} \exp (-k t)+\sqrt{k S c} \operatorname{erf} \sqrt{k t}\right] \\
& +a_{7}\left[\sqrt{\frac{\operatorname{Pr}}{\pi t}} \exp \left(-\frac{R t}{\operatorname{Pr}}\right)+\exp \left(a_{2} t\right) \sqrt{R+a_{2} \operatorname{Pr}} \operatorname{erf} \sqrt{\left(\frac{R}{\operatorname{Pr}}+a_{2}\right) t}\right]
\end{aligned}
$$




$$
\begin{aligned}
& -a_{5}\left[t \sqrt{k S c} \operatorname{erf}(\sqrt{k t})+\sqrt{\frac{t S c}{\pi}} \exp (-k t)+\frac{\sqrt{S c}}{2 \sqrt{k}} \operatorname{exf}(\sqrt{k t})\right], \\
& S h=t \sqrt{k S c} \operatorname{erf} \sqrt{k t}+\sqrt{\frac{t \operatorname{Pr}}{\pi}} \exp (-k t)+\frac{\sqrt{S c}}{2 \sqrt{k}} \operatorname{erf} \sqrt{k t} .
\end{aligned}
$$

\section{Results and Discussion}

To understand the mathematical model's physics, an analytical study has been conducted, presenting the results in the form of plots. The fluid properties such as velocity, concentration, and the rates of heat and mass transfer of the fluid as a result of the difference in the most significant non-dimensional parameters like magnetic parameter $(M)$, Dufour number $(D u)$, radiation parameter $(R)$, chemical reaction parameter $(k)$, Schmidt number $(S c)$, Prandtl number $(P r)$, thermal and solutal Grashof numbers $(G r \& G m)$ in Figures 2-11. To verify whether the obtained results are correct or not, a comparative study has been done when $B_{0}$ fixed to the fluid $(K=0)$ with the existing literature in the absence of some dimensionless quantities and found in good agreement.

The variations of $M, G m, D u, G r, S c$, and $t$ on the velocity profiles are presented from figures 2-7 for an exponentially accelerated plate in the presence of radiation and chemical reaction $K=0 \& 1$ by fixing other parameters $S c=2.01, R=10, \operatorname{Pr}=0.71, t=0.4, k=1, D u=0.03$, $\alpha=60^{\circ}, \varphi=30^{\circ}, M=5, a_{0}=0.5$. The outcome of the magnetic field on velocity profiles for $K=0$ and $K=1$ are discussed in Figures 2 and 3. It is observed that the velocity profiles reduce with an increase in the magnetic parameter when cooling and heating the plate. Applying an inclined magnetic field to an electric conductor fluid causes a drag force (Lorentz force). The effect of Dufour number on the velocity profiles is depicted in Figures. 4 and 5 for $K=0$ and $K=1$. It is observed that, in the case of plate cooling, the velocity reduces with the increase of the Dufour number, the trend is reversed when heating the plate. Figures 6 and 7 disclose the effects of time $t$ on the velocity profiles for $K=0$ and $K=1$. From these figures, it is seen that the velocity profiles rise as the plate is cooled and heated.

The variation of different physical quantities on temperature profiles are shown in Figures 8-10. Figure 8 shows the influence of Dufour number on temperature distributions, noticing that diffusional thermal effects significantly impact the liquid's temperature, and the fluid temperature decreases as the Dufour number increases. The effect of the thermal radiation $(R)$ parameter on the temperature is shown in Figure 9. From there, it is seen that the radiation parameter reduces the temperature of the liquid. The variations of Prandtl number $(\mathrm{Pr})$ illustrated on the temperature field in figure 10 reveal that as Princreases, the temperature decreases. This is because the thickness of the thermal boundary layer decreases. The variations of Schmidt number $(S c)$, by considering hydrogen $(S c=0.22)$, water $(S c=0.60)$, and oxygen $(S c=0.75)$ and the time $(t)$ on concentration profiles are depicted in Figure 11. Figure 11 shows that the concentration regularly rises for hydrogen and accumulates oxygen in contrast to the water vapor. Thus water vapor can be used to maintain the concentration profiles, and hydrogen can be used to maintain a strong concentration field.

The concentration profile decreases with an increase in $k$, as it is observed in Figure 12. Figure 13 shows the Nusselt number versus time $t$. 


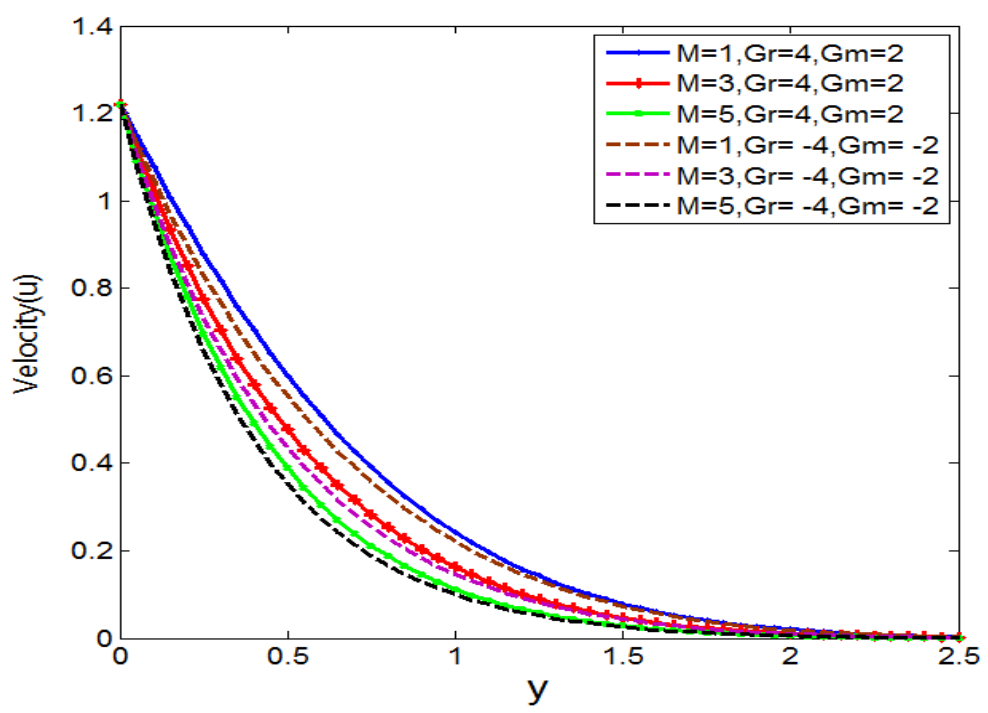

Figure 2. When $K=0$, the velocity variations for distinct $M$ values.

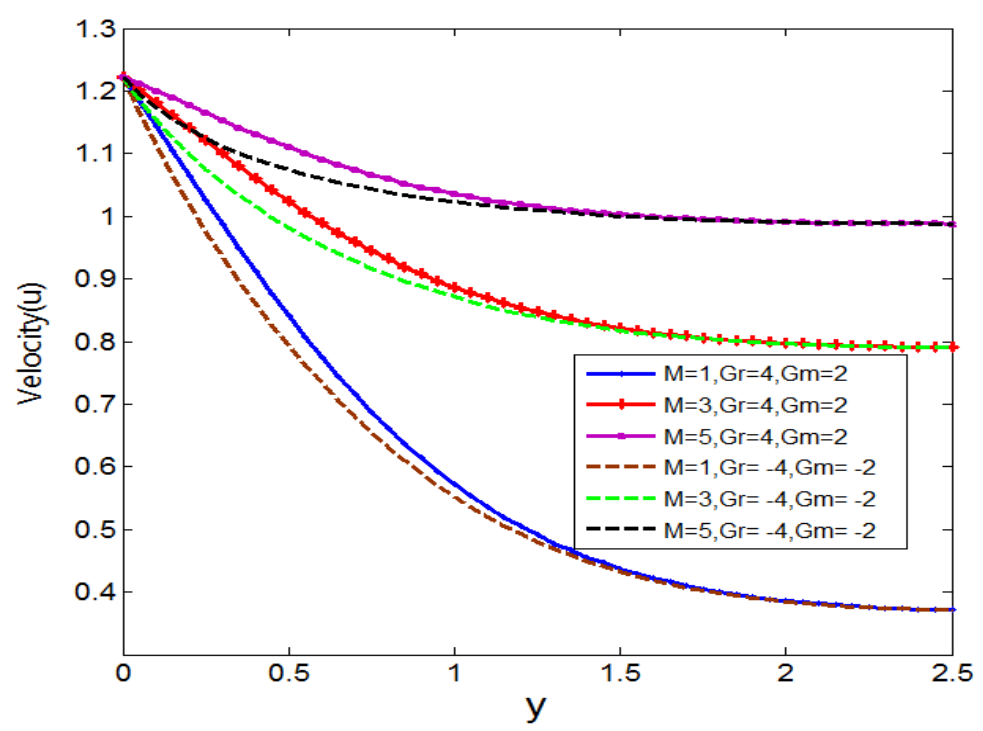

Figure 3. When $K=1$, the velocity variations for distinct $M$ values.

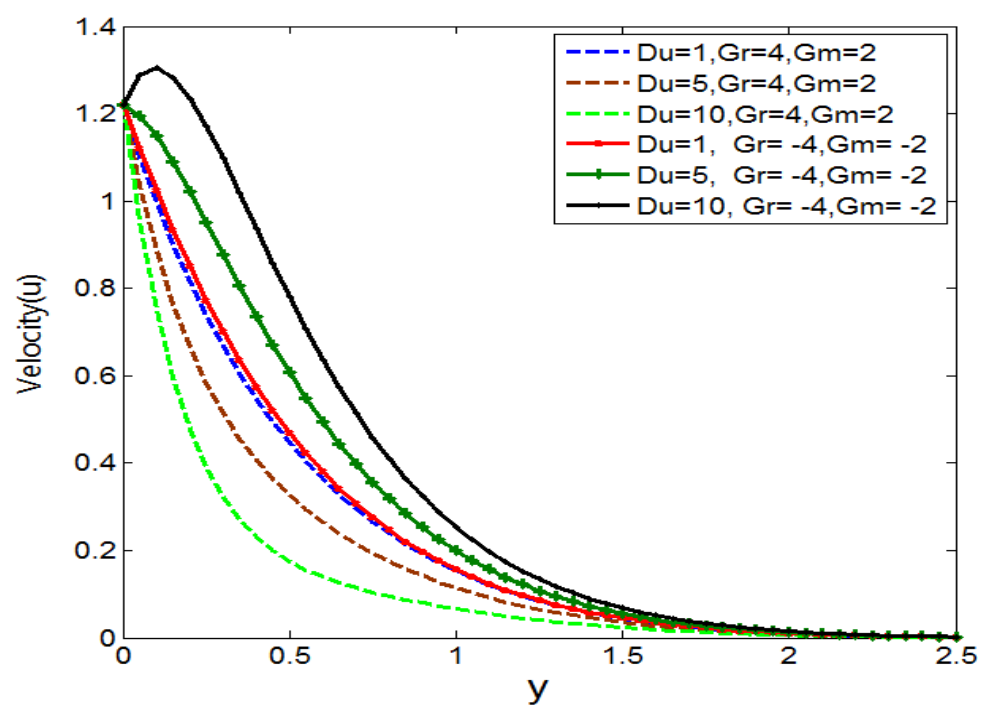

Figure 4. When $K=0$, the velocity variations for distinct $D u$ values.

The Nusselt number rises with increasing $R$ values for both air $(\operatorname{Pr}=0.71)$ and water $(\operatorname{Pr}=7.0)$, and it is seen that the rate of heat transfer with the water plate is high compared to air. This is because modest values $\operatorname{Pr}$ are equivalent to an increase in heat transmission and 
therefore, can spread much faster than higher Pr values. Figure 14 represents the Sherwood number versus time $t$. The Sherwood number rises with the increase of $S c$.

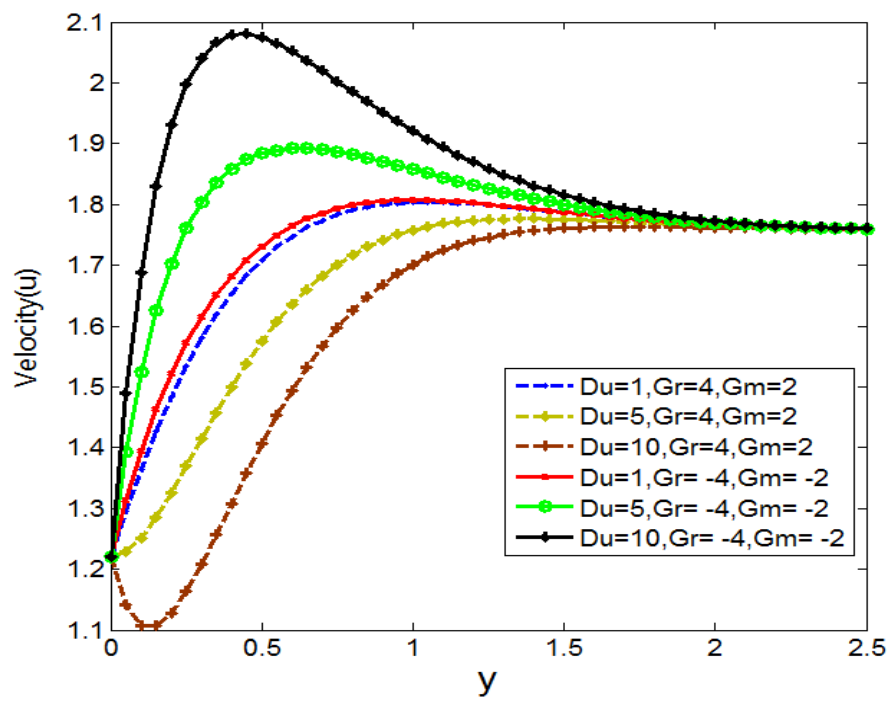

Figure 5. When $K=1$, the velocity variations for distinct $D u$ values.

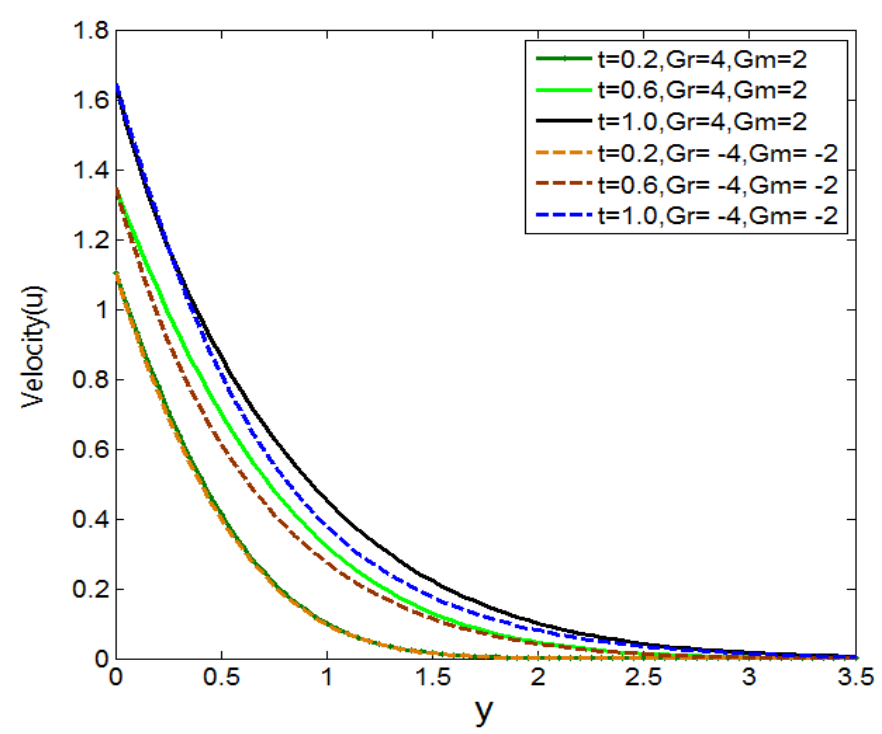

Figure 6. When $K=0$, the velocity variations for distinct $t$ values.

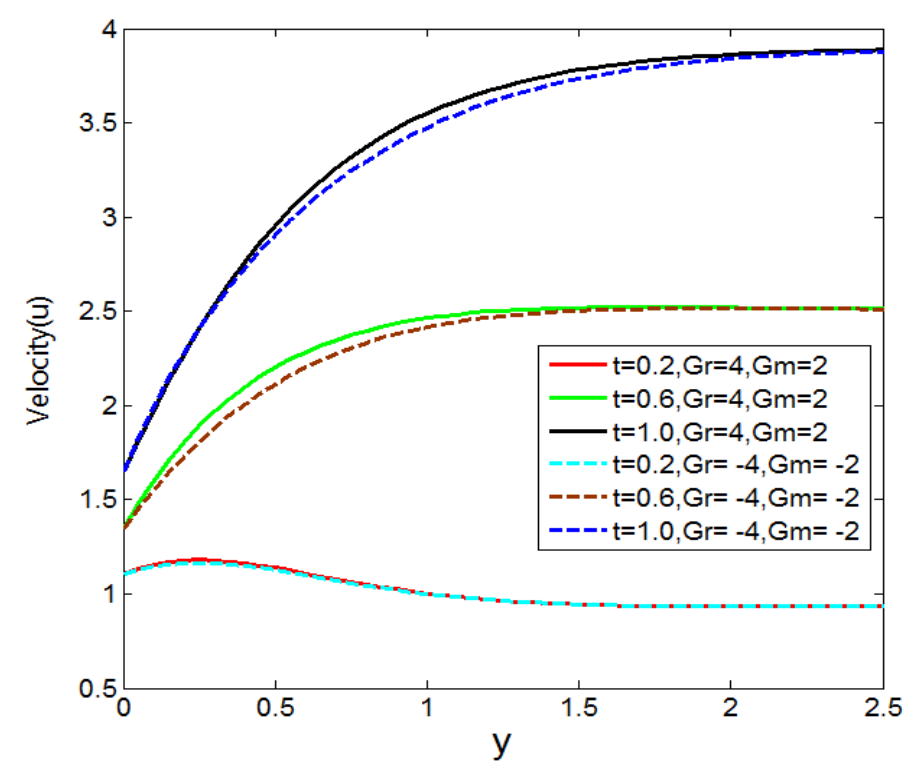

Figure 7. When $K=1$, the velocity variations for distinct $t$ values. 


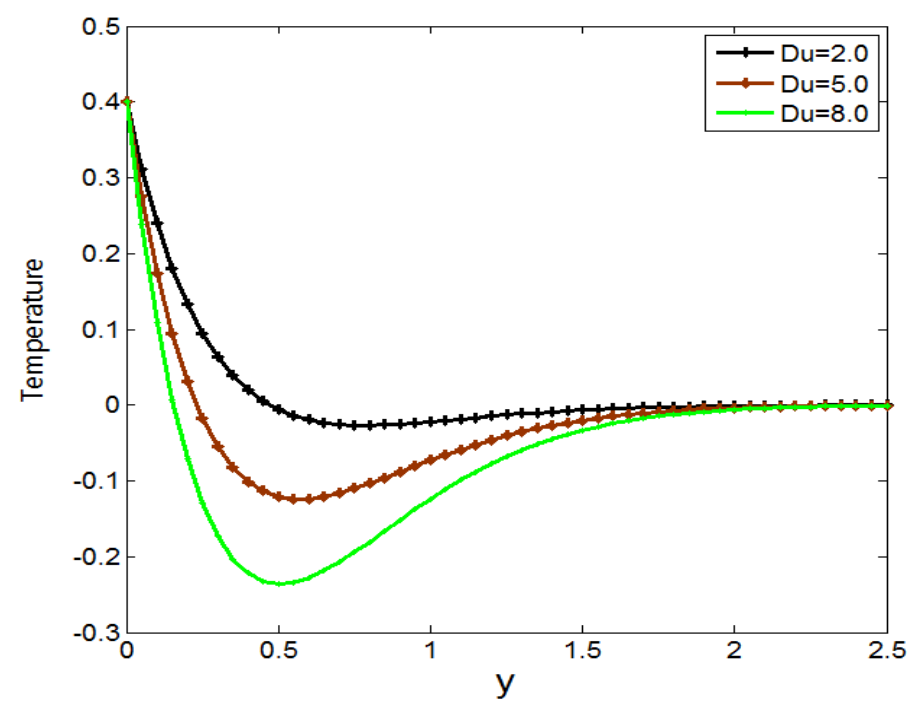

Figure 8. Temperature variations for distinct $D u$ values, when $S c=2.1, R=10, \operatorname{Pr}=0.71, t=0.4, k=0.5$.

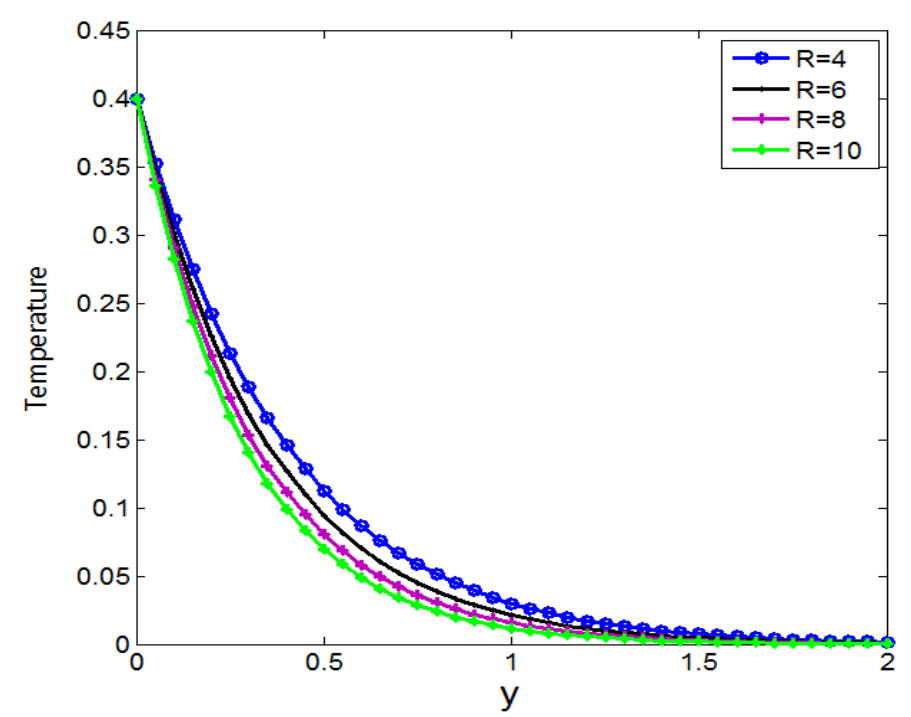

Figure 9. Temperature variations for distinct $R$ values, when $S c=2.1, D u=0.03, \operatorname{Pr}=0.71, t=0.4, k=0.5$.

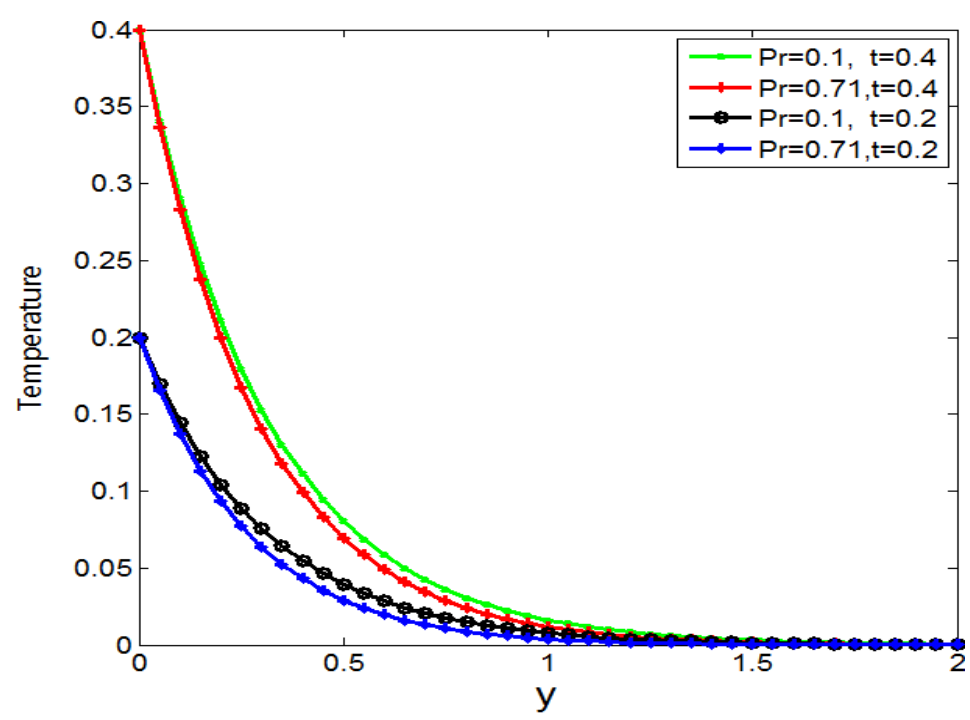

Figure 10. Temperature variations for distinct $\operatorname{Pr}$ and $t$ values, 


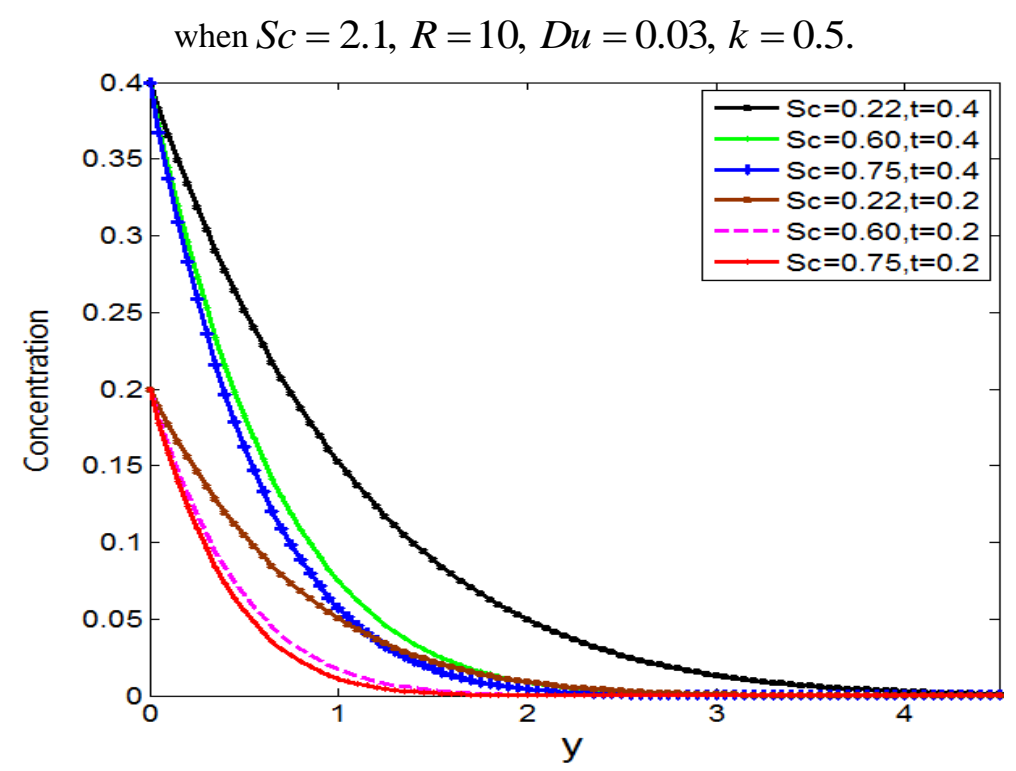

Figure 11. Concentration variations for distinct $S c$ and $t$ values, when $k=0.5$.

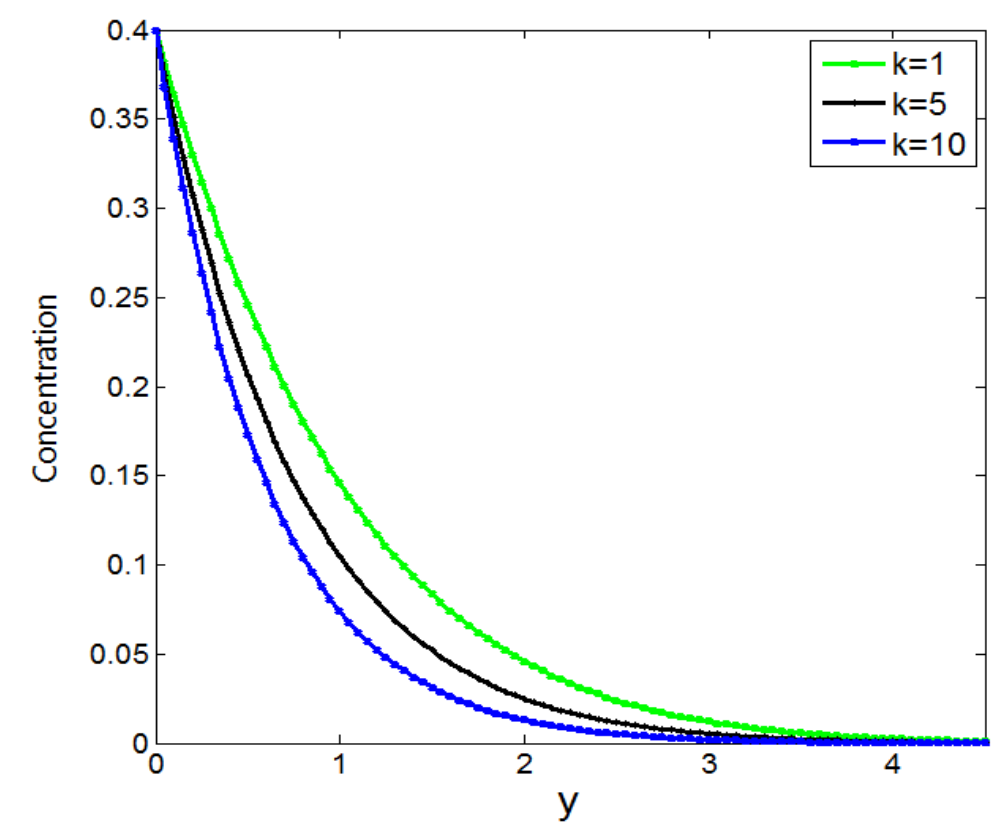

Figure 12. Concentration variations for distinct $k$ values, when $S c=0.22, t=0.4$.

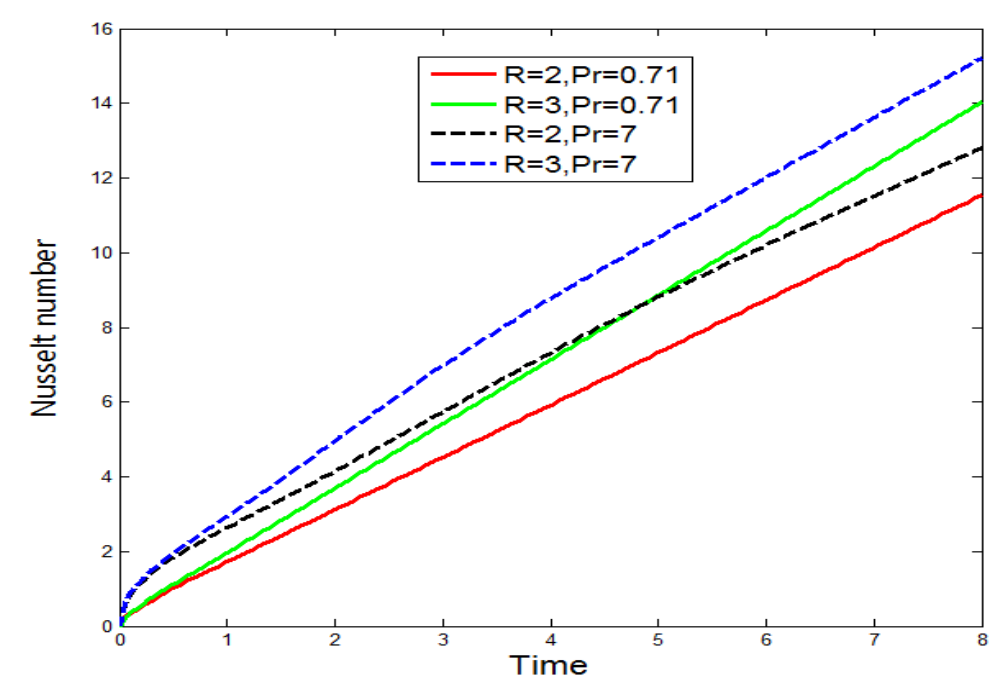

Figure 13. Nusselt number for distinct $R$ and $\operatorname{Pr}$ values, when $S c=2.1, D u=0.03, R=10, k=0.5$. 


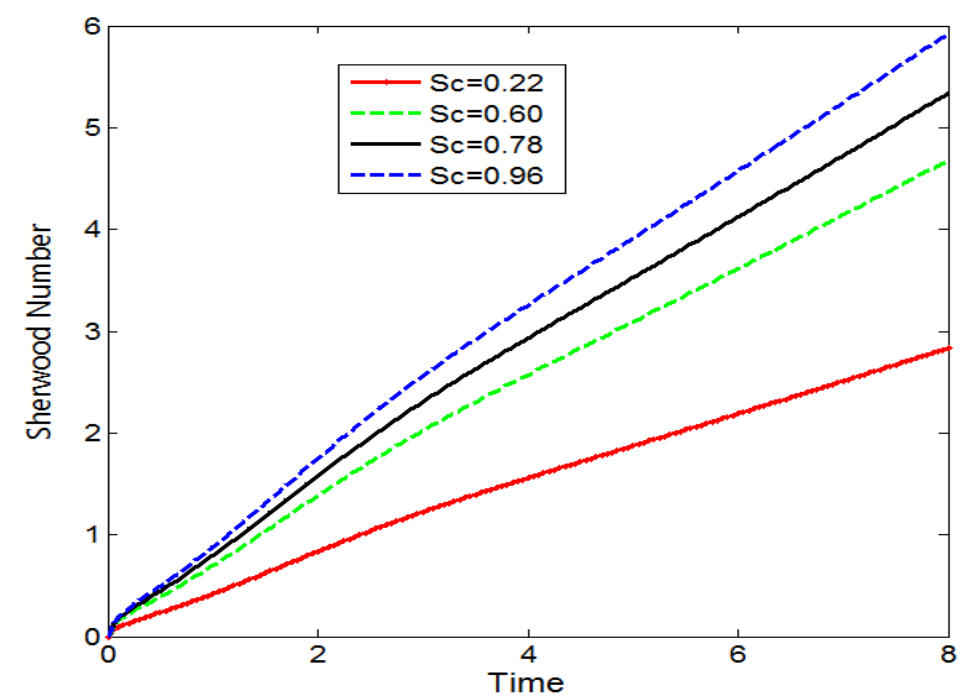

Figure 14. Sherwood number for distinct $S c$ values, when $\operatorname{Pr}=0.71$ and $k=0.5$.

Table 1 shows the change in the velocity profile for various values of $R$. It is observed that velocity reduces when heating and cooling the plate for $K=0$. The opposite trend is seen for $K=1$.

Table 1. Velocity distributions for various values of $R$, when $K=0,1$.

\begin{tabular}{|c|c|c|c|c|c|c|c|}
\hline & \multicolumn{4}{|c|}{$K=0$} & \multicolumn{3}{|c|}{$K=1$} \\
\hline & $y$ & $R=1$ & $R=5$ & $R=10$ & $R=1$ & $R=5$ & $R=10$ \\
\hline \multirow{6}{*}{ Cooling of the plate } & 0.0000 & 1.2214 & 1.2214 & 1.2214 & 1.2214 & 1.2214 & 1.2214 \\
\hline & 0.2000 & 0.9385 & 0.9335 & 0.9290 & 1.5323 & 1.5272 & 1.5228 \\
\hline & 0.4000 & 0.6995 & 0.6917 & 0.6856 & 1.7046 & 1.6968 & 1.6907 \\
\hline & 0.6000 & 0.5055 & 0.4972 & 0.4912 & 1.7890 & 1.7806 & 1.7746 \\
\hline & 0.8000 & 0.3540 & 0.3464 & 0.3413 & 1.8209 & 1.8133 & 1.8083 \\
\hline & 1.0000 & 0.2398 & 0.2336 & 0.2298 & 1.8244 & 1.8182 & 1.8143 \\
\hline \multirow{6}{*}{ Heating of the plate } & 0.0000 & 1.2214 & 1.2214 & 1.2214 & 1.2214 & 1.2214 & 1.2214 \\
\hline & 0.2000 & 0.8742 & 0.8793 & 0.8837 & 1.4679 & 1.4730 & 1.4774 \\
\hline & 0.4000 & 0.6211 & 0.6289 & 0.6350 & 1.6262 & 1.6339 & 1.6401 \\
\hline & 0.6000 & 0.4353 & 0.4437 & 0.4497 & 1.7187 & 1.7271 & 1.7331 \\
\hline & 0.8000 & 0.2991 & 0.3067 & 0.3118 & 1.7661 & 1.7737 & 1.7787 \\
\hline & 1.0000 & 0.2005 & 0.2067 & 0.2105 & 1.7850 & 1.7912 & 1.7950 \\
\hline
\end{tabular}

\section{Conclusions}

In this article, the effects of diffusion-thermo, thermal radiation, and chemical reaction are studied on unsteady, free convective MHD fluid passing through an inclined plate with variable surface conditions and concentration diffusion change under a coaxial magnetic field, when $K=0$ and $K=1$. A theoretical study has been conducted using Laplace transform method without any restriction in order to get the solution of the problem. The fluid properties for various flow controlling parameters like $M, R, S c, P r, D u, G r, G m$ are analyzed through graphs. A comparison is made with published results in the absence of some non-dimensional parameters. The following conclusions are drawn to the present study: (i) in case of cooling of the plate, the velocity decreases with an increasing $M$ and $D u$ values, and an opposite trend is observed in the case of heating the plate; (ii) the temperature profiles decrease significantly with increasing values of $D u, P r$, and $R$; (iii) the concentration profiles decrease significantly with increasing values of $S c$ and $k$; (iv) the Nusselt number increases with increasing $R$ values, and Sherwood number increases with the increase of $S c$. 


\section{Funding}

This research received no external funding.

\section{Acknowledgments}

The authors would like to thank the anonymous reviewers for their valuable comments and suggestions to improve the paper's quality.

\section{Conflicts of Interest}

The authors declare no conflict of interest.

\section{References}

1. Kao, T.-T. Laminar free convective heat transfer response along a vertical flat plate with step jump in surface temperature. Letters in Heat and Mass Transfer 1975, 2, 419-428, https://doi.org/10.1016/00944548(75)90008-9.

2. Raptis, A.; Singh, A.K. MHD free convection flow past an accelerated vertical plate. International Communications in Heat and Mass Transfer 1983, 10, 313-321, http://dx.doi.org/10.1016/07351933(83)90016-7.

3. Tokis, J.N. A class of exact solutions of the unsteady magnetohydrodynamic free-convection flows. Astrophysics and Space Science 1985, 112, 413-422, http://dx.doi.org/10.1007/bf00653524.

4. Prasad, k.V.; Vajravelu, K. MHD mixed convection heat transfer in a vertical channel with temperaturedependent transport properties. Journal of Applied Fluid Mechanics 2015, 8, 693-701, http://dx.doi.org/10.18869/acadpub.jafm.67.223.21562.

5. Sheikholeslami, M.; Rashidi, M.M.; Ganji, D.D. Effect of non-uniform magnetic field on forced convection heat transfer of Fe3O4-water nanofluid. Computer Methods in Applied Mechanics and Engineering 2015, 294, 299-312, http://dx.doi.org/10.1016/j.cma.2015.06.010.

6. Rashidi, M.M.; Nasiri, M.; Khezerloo, M.; Laraqi, N. Numerical investigation of magnetic field effect on mixed convection heat transfer of nanofluid in a channel with sinusoidal walls. J. Magn. Magn. Mater. 2016, 401, 159-168, http://dx.doi.org/10.1016/j.jmmm.2015.10.034.

7. Shateyi, S. Heat and mass transfer for natural convection MHD flow over a permeable moving vertical plate with convective boundary condition in the presence of viscous dissipation. AIP Conf. Proc. 2017, 1863, 560075, https://doi.org/10.5098/hmt.9.7.

8. Lawal, K.K.; Jibril, H.M. Impact of relative motion of a magnetic field on unsteady magnetohydrodynamic natural convection flow with a constant heat source/sink. Heat Transfer 2021, 50, 487-507, https://doi.org/10.1002/htj.21888.

9. Ilias, M.R.; Ismail, N.S.a.; Ab Raji, N.H.; Rawi, N.A.; Shafie, S. Unsteady aligned MHD boundary layer flow and heat transfer of a magnetic nanofluids past an inclined plate. International Journal of Mechanical Engineering and Robotics Research 2020, 9, https://doi.org/10.18178/ijmerr.9.2.197-206.

10. Fenuga, O.J.; Aroloye, S.J.; Salawu, S.O. Mathematical model and solution for an unsteady MHD fourth grade fluid flow over a vertical plate in a porous medium with magnetic field and suction/injection effects. Journal of Scientific Research 2020, 12, 485-498, https://doi.org/10.3329/jsr.v12i4.45551.

11. Rama Prasad, J.L.; Balamurugan, K.S.; Varma, S.V.K. Aligned magnetic field effect on unsteady MHD double diffusive free convection flow of Kuvshinski fluid past an inclined moving porous plate. In Proceedings of Advances in Fluid Dynamics, Singapore, 2021; 255-262, https://doi.org/10.1007/978-981-154308-1.

12. Bilal, M.; Nazeer, M. Numerical analysis for the non-Newtonian flow over stratified stretching/shrinking inclined sheet with the aligned magnetic field and nonlinear convection. Archive of Applied Mechanics 2020, https://doi.org/10.1007/s00419-020-01798-w.

13. Alam, M.S.; Rahman, M.M. Dufour and Soret effects on mixed convection flow past a vertical porous flat plate with variable suction. Nonlinear Anal. Model. Control 2006, 11, 3-12, https://doi.org/10.15388/na.2006.11.1.14761. 
14. Suresh Babu, R.; Kumar, B.R.; Dinesh, P.A. Soret and Dufour effects on MHD mixed convection flow over a vertical plate with variable fluid properties. Defect and Diffusion Forum 2018, 389, 1-17, https://doi.org/10.4028/www.scientific.net/ddf.389.1.

15. Reddy, G.S.; Reddy, S.K.; Prasad, P.D.; Varma, S.V. Diffusion-thermo and aligned magnetic field effects on free convection on flow past an inclined porous plate with first order chemical reaction. IOSR Journal of Electrical and Electronics Engineering, 2020, 12, 25-33, https://doi.org/10.9790/1676-1203022533.

16. Rao, T.S.N. Effect of thermo diffusion on mass and heat transfer flow on convective viscous electrically conducting fluid through a porous medium bounded by a semi-infinite vertical plate with variable electrically conductivity diffusion thermo chemical reaction. International Journal of Engineering and Advanced Technology, 2019, 9, 284-287, https://doi.org/10.35940/ijeat.a1063.1291s52019.

17. Ramalingeswara Rao, S.; Vidyasagar, G.; Deekshitulu, G.V.S.R. Unsteady MHD free convection Casson fluid flow past an exponentially accelerated infinite vertical porous plate through porous medium in the presence of radiation absorption with heat generation/absorption. Materials Today: Proceedings 2020, https://doi.org/10.1016/j.matpr.2020.07.554.

18. Mahato, R.; Das, M. Effect of suction/blowing on heat-absorbing unsteady radiative Casson fluid past a semiinfinite flat plate with conjugate heating and inclined magnetic field. Pramana 2020, 94, 127, https://doi.org/10.1007/s12043-020-01990-1.

19. Omamoke, E.; Amos, E.; Bunonyo, K.W. Radiation and heat source effects on MHD free convection flow over an inclined porous plate in the presence of viscous dissipation. American Journal of Applied Mathematics 2020, 8, 190-206, https://doi.org/10.11648/j.ajam.20200804.14.

20. Lavanya, S.; Devika, B.; Chenna Kesavaiah, D. Radiation effect on unsteady free convective MHD flow of a viscoelastic fluid past a tilted porous plate with heat source. Journal of Xidian University 2020, 14, 22982312, https://doi.org/10.37896/jxu14.4/256.

21. Suresh Babu, R.; Kumar, B.R.; Mallikarjuna, B.; Dinesh, P.A. Effects of variable fluid properties on MHD non-Darcy convective flow from vertical plate with heat generation and chemical reaction. Defect and Diffusion Forum 2018, 388, 190-203, https://doi.org/10.4028/www.scientific.net/ddf.388.190.

22. Krishna, C.M.; Reddy, G.V.; Ramakrishna, S.B.; Mamatha, S.U.; Raju, C.S.K. Mixed convective conditions on natural convection of MHD Blasius and Sakiadis flows with variable properties and nonlinear chemical reaction. Biointerface Research in Applied Chemistry 2020, 11, 8854-8874, https://doi.org/10.33263/briac112.88548874.

23. Lalitha, P.; Varma, S.V.K. Thermal radiation and chemical reaction effects on unsteady laminar hydro magnetic free convective flow of oldroyd fluid through porous medium along a moving vertical plate with thermal diffusion. International Journal of Mathematics Trends and Technology 2018, 51, 43-51, https://doi.org/10.14445/22315373/ijmtt-v51p506.

24. Suresh Babu, R.; Kumar, B.R.; Makinde, O.D. Chemical reaction and thermal radiation effects on MHD mixed convection over a vertical plate with variable fluid properties. Defect and Diffusion Forum 2018, 387, 332-342, https://doi.org/10.4028/www.scientific.net/ddf.387.332.

25. Endalew, M.F.; Nayak, A.; Sarkar, S. Flow past an oscillating slanted plate under the effects of inclined magnetic field, radiation, chemical reaction, and time-varying temperature and concentration. International Journal of Fluid Mechanics Research 2020, 4, https://doi.org/10.1615/interjfluidmechres.2020026987.

26. Sarma, D.; Pandit, K.K. Effects of thermal radiation and chemical reaction on steady MHD mixed convective flow over a vertical porous plate with induced magnetic field. International journal of fluid Mechanics Research 2015, 42, https://doi.org/10.1615/interjfluidmechres.v42.i4.30.

27. Mohan, S.R.; Reddy, G.V.; Varma, S.V.K. Soret and aligned magnetic field effects on an unsteady MHD free convection Casson fluid flow past an exponentially infinite vertical plate through porous medium in the presence of thermal radiation, chemical reaction and heat source or sink. Advanced Science, Engineering and Medicine 2019, 11, 336-345, https://doi.org/10.1166/asem.2019.2355.

28. Bati Kejela, S.; Daba Firdi, M. Analytical analysis of effects of buoyancy, internal heat generation, magnetic field, and thermal radiation on a boundary layer over a vertical plate with a convective surface boundary condition. International Journal of Differential Equations 2020, 2020, 8890510, https://doi.org/10.1155/2020/8890510.

29. Raju, R.S.; Reddy, G.J.; Kumar, M.A.; Gorla, R.S.R. Unsteady MHD chemically reacting fluid flow past an inclined vertical permeable moving plate. International Journal of Fluid Mechanics Research 2020, 47, 191215, https://doi.org/10.1615/interjfluidmechres.2020028808. 
30. Rao, S.R.; Vidyasagar, G.; Deekshitulu, G.V.S.R. Aligned magnetic field effects on an unsteady MHD free convective Laminar flow past an exponentially infinite vertical porous plate in the presence of radiation absorption, chemical reaction and heat generation. Strad Research 2020, 7, 999-1011, https://doi.org/10.37896/sr7.10/106.

31. Mohan, S.R.; Reddy, G.V.; Varma, S.V.K. Effects of chemical reaction, radiation and aligned magnetic field on unsteady MHD Casson fluid flow past a moving an infinite vertical plate through porous medium in presence of heat absorption. i-Manager's Journal on Future Engineering and Technology 2019, 14, 47, https://doi.org/10.26634/jfet.14.4.14712.

32. Omamoke, E.; Amos, E. Chemical Reaction, radiation and heat source effects on unsteady MHD blood flow over a horizontal porous surface in the presence of an inclined magnetic field. International Journal of Scientific and Engineering Research 2020, 11, 1187-1192, https://doi.org/10.14299/ijser.2020.04.02. 